Ann. Zootech., I974, 23 (2), I49-I60.

\title{
INFLUENCE DES CONDITIONS D'AGGLOMÉRATION SUR QUELQUES CARACTÉRISTIQUES PHYSICO-CHIMIQUES D'UN ALIMENT POUR PORCELET
}

\author{
J. P. MELCION, P. VAISSADE* \\ Paulette VALDEBOUZE et G. VIROBEN \\ avec la collaboration technique d'Édith BERGERON, \\ Georgette Gianni, M. Hervio et Françoise Kozlowski \\ Laboratoire de Technologie des Aliments des Animaux, \\ * Station de Recherches sur l'Élevage des Porcs, \\ Centre national de Recherches zootechniques, I. N. R. A., \\ 78350 Jouy en Josas
}

RÉSUMÉ

Un aliment pour porcelet a été aggloméré à une échelle semi-industrielle selon 3 modalités différentes : à sec, à l'eau ou avec vapeur. Nous avons tenté d'évaluer les modifications subies par certains de ses composants (lysine, glucides, amidon et vitamine A), et de définir les caractéristiques physiques des agglomérés obtenus en fonction des paramètres du pressage.

Dans nos conditions expérimentales, nous n'avons mis en évidence aucune réduction significative de la fraction « disponible » de la lysine. Par contre, la vitamine A, les glucides réducteurs totaux et la sensibilité de l'amidon à l'attaque amylasique sont affectés par les conditions les plus sévères (pressage à sec). L'action combinée de la pression et de la chaleur semble déterminante, en particulier sur l'amidon, alors que la chaleur seule ou la pression accompagnée d'une faible élévation de température n'ont pratiquement pas d'effet sur les critères physico-chimiques retenus.

\section{INTRODUCTION}

L'intérêt de l'agglomération d'un aliment composé est bien connu, tant par ses aspects technologiques (densité accrue, facilité de manutention et de stockage) que nutritionnels, décrits par de nombreux auteurs, pour le porcelet (NELSON et CATRON,

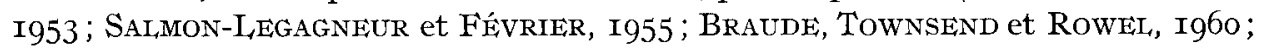

Annales de Zootechnie. - I974. 
Aumaitre et Salmon-Legagneur, I96I ; LAWREnCe, I97I), le porc à l'engrais (Jensen, I966; VAnschoubroek, Coucke et VAn Spaendonck, I97I) et le poulet de chair (Allired, Jensen et McGinnis, I957; Jensen et al., ig62; CAlet, I965).

Les forces de compression intenses mises en jeu provoquent l'apparition d'une structure physique nouvelle qu'il importe de caractériser (DREVET, I970) ; jointes à une élévation brutale de la température, elles peuvent déclencher dans le produit de nombreuses réactions physico-chimiques, et influer notamment sur l'état de dégradation de l'amidon, l'altération des vitamines et la disponibilité de la lysine. Ainsi, à la suite de MAILLARD (IgI2) de nombreux auteurs ont montré qu'une partie de la lysine liée aux protéines d'un aliment peut être rendue indisponible par suite de la liaison des groupes aminés terminaux de cet acide aminé avec d'autres composés particulièrement réactifs, tels que les fonctions aldéhydiques des sucres réducteurs. Cette réaction (ADRIAN et FAVIER, I96I; ADRIAN, I972) est favorisée par une faible hydratation du milieu et une élévation de température même modeste. Par ailleurs, l'agglomération de l'aliment peut être assimilée à un traitement hydrothermique de la fraction amylacée (Guilbot et Mercier, ig62; Mercier et Guilbot, i962; Aumaitre, I972) dont l'état se trouve plus ou moins modifié.

Enfin, le pressage paraît retentir également sur la détérioration de certains additifs (STOCKSTAd et al., I952 ; Salmon-LEGagneur, Aumaitre et Jouandet, I968) et vitamines de l'aliment (BIERER et VICKERS, I958; WorNICK, r959 et I968; FraNÇOIS, I960 ; VALDEBOUZE et I,EVY, I972), mais les résultats sont très variables selon les auteurs.

\section{MATÉRIELS ET MÉTHODES}

\section{г. - Fabrication de l'aliment}

L'aliment choisi, dont la composition est donnée dans les tableaux I et 2 , est un aliment classique pour porcelet (type "Sevrage 35 jours ") riche en protéines et en glucides simples (saccharose et lactose). Sa teneur en vitamine A enrobée (concentrat à 50 ooo UI/g) est de I ooo ooo UT/ Ioo kg, dans le premier essai (pressage à l'eau), du double dans les suivants.

TABL,EAU I

Composition de l'aliment expérimental (en p. roo)

(type "Sevrage 35 jours")

\begin{tabular}{|c|c|}
\hline Orge $\ldots \ldots \ldots \ldots \ldots \ldots \ldots$ & 27 \\
\hline Soja $\ldots \ldots \ldots \ldots \ldots \ldots \ldots \ldots \ldots \ldots \ldots$ & 20 \\
\hline Farine de poisson $\ldots \ldots \ldots \ldots \ldots \ldots \ldots \ldots \ldots$ & 6 \\
\hline Lait spray $\ldots \ldots \ldots \ldots \ldots \ldots \ldots \ldots \ldots \ldots \ldots$ & 5 \\
\hline Huile de maïs $\ldots \ldots \ldots \ldots \ldots \ldots \ldots \ldots \ldots \ldots \ldots$ & 3 \\
\hline 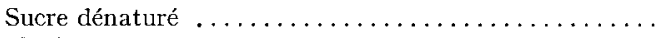 & 5 \\
\hline 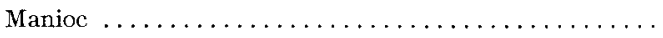 & 30 \\
\hline C. M. V. $\ldots \ldots \ldots \ldots \ldots \ldots \ldots \ldots \ldots \ldots \ldots \ldots \ldots$ & 3,5 \\
\hline \multirow[t]{2}{*}{ 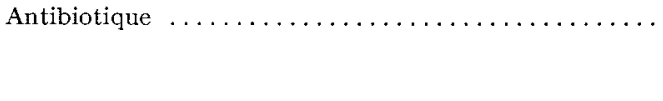 } & 0,5 \\
\hline & 100 \\
\hline
\end{tabular}


TABLEAU 2

Analyse fourragère de l'aliment utilisé (en p. Ioo du produit brut)

(échantillons moyens)

\begin{tabular}{|c|c|c|}
\hline & Farine & Agglomérés \\
\hline Humidité & 10,6 & 9,8 \\
\hline Matière sèche & $89,4_{4}^{\prime}$ & 90,2 \\
\hline Matières minérales $\ldots \ldots \ldots \ldots \ldots$. & 6,0 & 5,9 \\
\hline Matières cellulosiques $\ldots \ldots \ldots \ldots$ & 2,9 & 3,6 \\
\hline Matières azotées totales . . . . . . . & 20,1 & 19,8 \\
\hline Matières grasses . . . . . . . . . . & 4,2 & 4,1 \\
\hline
\end{tabular}

Après broyage et mélange effectués dans des conditions semi-industrielles, l'aliment est aggloméré dans une presse Gondard VT 400 de $100 \mathrm{CV}$ à filière tournante d'axe horizontal, de $40 \mathrm{~mm}$ d'épaisseur et de $2,5 \mathrm{~mm}$ de diamètre de perforations, selon 3 modalités différentes:

- addition d'eau (4 p. roo) ;

- injection de vapeur (soit I à I, 2 P. Ioo d'eau) ;

- à sec ;

en 3 séries successives de 2 tonnes d'aliment. Pour une même série, les déterminations et contrôles ne portent que sur la deuxième tonne fabriquée de façon à laisser s'instaurer dans la machine un régime d'équilibre. Les mesures sont effectuées sur les agglomérés par comparaison avec la farine correspondante prise comme témoin.

\section{2. - Contrôle de la fabrication}

Les conditions d'agglomération (température de pressage et de refroidissement, taux d'humidité du produit à l'entrée et à la sortie de la presse) influent sur le rendement de l'opération (débit horaire et énergie électrique consommée) et sont déterminées de la manière suivante :

- les températures de la farine, de l'aggloméré à la sortie de la presse et dans le refroidisseur sont repérées par des sondes à thermocouples, et la puissance mesurée par un thermoconvertisseur : ces capteurs sont reliés à un appareil enregistreur Meci ( I point par minute) ;

-- l'humidité de l'aliment est dosée à l'étuve infra-rouge à $80^{\circ} \mathrm{C}$ jusqu'à poids constant.

\section{3. - Échantillonnage et analyses}

Les farines et les agglomérés sont prélevés à l'entrée et à la sortie de la filiève, à un intervalle de 2 secondes correspondant au temps de passage, repéré au préalable à l'aide d'un marqueur constitué de maïs jaune pur.

\section{Échantillonnage.}

Ces prélèvements sont effectués toutes les deux minutes sur I tonne d'aliment, soit I 2 prises d'essai de I $\mathrm{kg}$ sur lesquelles sont analysés les glucides totaux et la lysine disponible. Les glucides alcoolosolubles, ainsi que la vitesse d'amylolyse sont déterminés sur 3 échantillons formés à partir des précédents, tandis que la mesure des caractéristiques physiques des agglomérés s'effectue sur un échantillon moyen unique.

Pour le dosage de la vitamine A, sont prélevées à part, mais dans les mêmes conditions, 5 prises d'essai d'environ roo g par tonne d'aliment, qui sont analysées le jour même.

\section{Caractéristiques physiques.}

La dureté, définie par la résistance maximale à la compression radiale d'un ensemble d'agglomérés de même diamètre et de longueurs connues (DELORT-LAVAL et DrEVET, r970), est mesurée à l'aide d'un compressomètre Lhomargy : le résultat, exprimé en bars par $\mathrm{cm}$ de longueur d'aggloméré, est la moyenne des mesures effectuées sur I 2 échantillons de ro agglomérés. 
La friabilité est exprimée par le pourcentage moyen $(n=6)$ de particules fines obtenues en Io minutes sur 400 g d'aliment aggloméré dans un appareil à caissons rotatifs tournant à $5^{\circ} \mathrm{tr} / \mathrm{mn}$ (Pfost et Allen, ig63; Schultz, ig65).

Analyse chimique.

a) Lysine totale.

Après hydrolyse des échantillons par l'acide chlorhycirique $6 \mathrm{~N}\left(24\right.$ heures - I $\left.30^{\circ} \mathrm{C}\right)$, la lysine totale a été séparée et dosée par chromatographie sur colonne (Moore, Spackman, Stern, I958) à l'aide d'un analyseur automatique Phoenix.

b) Lysine " disponible".

Pour la détermination de la lysine " disponible ", nous avons choisi la méthode de CARPENTER (I960) en raison de sa facilité d'adaptation aux dosages en série, malgré son imprécision sur un produit riche en glucides, et notamment en amidon. La méthode de RoAch, SANDERSON et Williams (1967) aurait donné des valeurs plus exactes, mais elle ne répond pas aux critères de simplicité et rapidité habituellement exigées pour le choix d'une méthode.

Une seule formule d'aliment ayant été considérée, nous avons supposé constante la destruction partielle de la DNP-lysine au cours de l'hydrolyse, de façon à pouvoir surtout comparer entre eux les différents traitements subis par ce même aliment.

c) Glucides totaux et alcoolosolubles.

Les glucides totaux ont été dosés selon la méthode cuprimétrique, dérivée de celle de BERTRAND (hydrolyse de l'extrait aqueux en milieu chlorhydrique environ $\mathrm{N} / 4$, entre 70 et $75^{\circ} \mathrm{C}$ pendant 20 minutes). Par ailleurs, le dosage des glucides solubles dans l'alcool à 80 p. roo a été effectué par la méthode colorimétrique à l'anthrone utilisant le glucose comme référence.

d) Vitamine $A$.

La suppression de la chromatographie préalable a permis d'adapter au dosage en série la méthode mise au point par Françors et PIHET (I96I). Ce procédé surestime les teneurs réelles par suite de l'interférence de certaines substances, habituellement éliminées lors de la chromatographie. Comme dans le cas de la DNP-lysine, nous supposerons cette interférence sensiblement constante, tous les échantillons d'un même essai ayant été traités dans les mêmes conditions.

e) Etat de l'amidon.

La vitesse de l'hydrolyse de l'amidon contenu dans l'aliment (Mercier, 1968) est déterminée par la mesure automatique des glucides formés au cours de l'attaque d'un échantillon de produit dispersé dans une solution tampon et mis en présence de suc pancréatique de porcelet (VAISSADE, non publié). Quatre types d'aliments ont été retenus dans ce cas : l'aliment en farine pris comme témoin, l'aliment aggloméré à sec ou à la vapeur, l'aliment en farine après conditionnement à la vapeur. Trois mesures successives sont effectuées au cours de 24 heures d'hydrolyse en continu et comportent une dialyse des produits de la réaction à travers une membrane "Cuprophan $\mathrm{C}$ ". Les données sont exprimées en pourcentage d'amidon dégradé et transformé en glucides dialysables rapportés à une solution témoin de maltose titrée, en fonction du temps.

\section{RÉSULTATS}

Les données concernant le rendement de la presse et les caractéristiques physiques des agglomérés sont regroupées dans le tableau 3, les résultats des analyses chimiques dans les tableaux 4 et 5, ainsi qu'à la figure I.

\section{x. - Effet du mode de pressage sur les conditions d'agglo- mération et les caractéristiques physiques des agglomérés}

L'agglomération se traduit par une forte élévation de température, consécutive aux pressions très élevées (estimées à $400-3500 \mathrm{~kg} / \mathrm{cm}^{2}$ par FRIEDRICH, 1964) qui règnent dans une presse à filière. La température repérée dans un vase de DEWAR 
(tab1. 3) n'est qu'une température d'équilibre de l'aliment avec l'air d'une enceinte isolée et en réalité, la périphérie des agglomérés est portée à une température supérieure $\left(150-200^{\circ} \mathrm{C}\right)$ durant un temps très court.

\section{TABLEAU 3}

Influence du mode de pressage sur les conditions d'agglomération et les caractéristiques physiques des agglomérés

\begin{tabular}{|c|c|c|c|}
\hline & \multicolumn{3}{|c|}{ Mode de pressage } \\
\hline & eau & vapeur & sec \\
\hline \multicolumn{4}{|l|}{ Températures maximales $\left({ }^{\circ} \mathrm{C}\right)$} \\
\hline Entrée produit . . . . . . . & 24 & 20 & 14 \\
\hline Entrée vapeur $\ldots \ldots \ldots \ldots \ldots$ & $\ldots$ & 64 & 18 \\
\hline Entrée filière $\ldots \ldots \ldots \ldots \ldots$ & 25 & 43 & 18 \\
\hline Sortie filière $\ldots \ldots \ldots \ldots \ldots$ & 52 & 65 & 60 \\
\hline Agglomérés (Dewar) ....... & 58 & 73 & 77 \\
\hline Refroidisseur $\ldots \ldots \ldots \ldots \ldots$ & 34 & 43 & 36 \\
\hline \multicolumn{4}{|l|}{ Humidité (en p. 100) } \\
\hline Sortie mélangeuse $\ldots \ldots \ldots$ & 10,1 & 10,5 & $\ldots$ \\
\hline Entrée filière $\ldots \ldots \ldots \ldots \ldots$ & 14,1 & 11,6 & 11,7 \\
\hline Sortie filière $\ldots \ldots \ldots \ldots \ldots$ & 13,0 & 11,7 & 10,8 \\
\hline \multicolumn{4}{|l|}{ Débit horaire $(t / h)$} \\
\hline Brut $\ldots \ldots \ldots \ldots \ldots \ldots$ & 2,6 & 2,6 & 1,8 \\
\hline Matière sèche $\ldots \ldots \ldots \ldots \ldots$ & 2,8 & 2,9 & 2,0 \\
\hline $\begin{array}{l}\text { Énergie totale consommée } \\
\qquad(\mathrm{kWh} / \mathrm{t})\end{array}$ & 18,1 & 17,8 & 21,1 \\
\hline $\begin{array}{l}\text { Puissance moyenne absorbée } \\
\qquad(\mathrm{kW})\end{array}$ & 47,2 & 46,5 & 38,2 \\
\hline \multicolumn{4}{|l|}{ Caractéristiques physiques } \\
\hline Dureté $(\mathrm{bar} / \mathrm{cm}) \ldots \ldots \ldots$ & 2,8 & 4,2 & 7,7 \\
\hline Friabilité $(\%) \quad \ldots \ldots \ldots \ldots$ & 3,8 & 3,7 & 1,7 \\
\hline
\end{tabular}

* Non dosé.

En régime d'équilibre, le pressage à sec se différencie nettement (tabl. 3) du pressage à l'aide d'eau ou de vapeur. Le débit horaire est en effet moindre dans ce premier cas $(I, 8 \mathrm{t} / \mathrm{h}$ au lieu de 2,6$)$. On note un accroissement $(+\mathbf{I} 7 \mathrm{p}$. Ioo) de l'énergie totale consommée par la machine qui se traduit par un échauffement plus important des agglomérés à la sortie de la matrice : l'élévation de la température du produit au cours de la traversée de la filière ( $\Delta \theta=$ température des agglomérés à la sortie de la filière (Dewar) - température de la farine à l'entrée de la filière) est pratiquement doublée dans le cas du pressage à sec par rapport aux autres modes d'agglomération $\left(+59^{\circ} \mathrm{C}\right.$ contre $+30^{\circ} \mathrm{C}$ et $+33^{\circ} \mathrm{C}$ ). Il faut remarquer que cet échauffement est brutal puisqu'il 
s'effectue pendant la durée du transit de la matière à travers 1a matrice, soit environ 2 secondes. On peut concevoir que les calories et l'humidité apportées par la vapeur " préparent " la farine à l'agglomération par différents mécanismes (apparition de forces capillaires et de tension superficielle entre les particules, modification de la fraction amylacée) et diminuent ainsi l'apport dû aux seules forces de compression.

La dureté diminue proportionnellement à la quantité d'eau ajoutée (injection de vapeur, addition d'eau). Les phénomènes de soudure entre les particules semblent donc prédominer en l'absence d'eau : la cohésion de l'aggloméré s'en trouve renforcée et sa friabilité corrélativement réduite de façon significative.

\section{2. - Effet du mode de pressage sur les caractéristiques chimiques de l'aliment}

a. Lysine.

Comparées à la teneur en lysine de l'aliment, les valeurs enregistrées pour la lysine "disponible" (tabl. 4) sont faibles (environ 67 p. roo de la lysine totale), que l'aliment se présente sous forme de farine ou sous forme agglomérée. Cette diminution est due à la destruction partielle, signalée par de nombreux auteurs, de la DNP-lysine au cours de 1'hydrolyse, effectuée dans un milieu riche en amidon et en glucides.

\section{TABLEAU 4}

Influence du mode de pressage sur la disponibilité de la lysine de l'aliment et la stabilité de la vitamine $A$

\begin{tabular}{|c|c|c|c|c|}
\hline & \multirow{2}{*}{$\begin{array}{l}\text { Teneur } \\
\text { calculée }\end{array}$} & \multicolumn{3}{|c|}{ Mode de pressage } \\
\hline & & à l'eau & $\begin{array}{c}\text { avec } \\
\text { vapeur }\end{array}$ & à sec \\
\hline \multicolumn{5}{|l|}{ Lysine totale ( $\% \mathrm{MS})$} \\
\hline Farine $\ldots \ldots \ldots \ldots \ldots$ & 1,29 & 1,32 & 1,35 & 1,41 \\
\hline Agglomérés $\ldots \ldots \ldots \ldots$ & - & 1,30 & 1,29 & 1,38 \\
\hline \multicolumn{5}{|l|}{ Lysine disponible (\% MS) } \\
\hline Farine $\ldots \ldots \ldots \ldots \ldots$ & - & $0,89^{a}$ & $0,89^{a 1}$ & $0,93^{a 2}$ \\
\hline Agglomérés $\ldots \ldots \ldots \ldots$ & - & $\begin{array}{l}0,88^{a} \\
\text { NS }\end{array}$ & $\begin{array}{l}0,87^{a 1} \\
\text { NS }\end{array}$ & $\begin{array}{l}0,91^{a^{2}} \\
\mathrm{NS}\end{array}$ \\
\hline \multicolumn{5}{|l|}{ Vitamine A (UI/g MS) } \\
\hline $\begin{array}{l}\text { Farine } \ldots \ldots \ldots \ldots \ldots \\
\text { Agglomérés } \ldots \ldots \ldots \ldots \ldots\end{array}$ & $\stackrel{11,11}{-}$ & $\begin{array}{l}13,33^{a} \\
12,12^{a}\end{array}$ & & \\
\hline \multirow{3}{*}{$\begin{array}{l}\text { Farine ...... } \\
\text { Agglomérés }\end{array}$} & 22,22 & & $27,41^{a 1}$ & $26,34^{a 2}$ \\
\hline & - & & $25,56^{a 1}$ & $23,24^{b 2}$ \\
\hline & & NS & NS & $P<0,01$ \\
\hline
\end{tabular}

$N . B$. : Les valeurs affectées de la même lettre ne sont pas significativement différentes dans une même case. 
Les valeurs obtenues montrent que l'effet du pressage sur la teneur en lysine " disponible " de l'aliment aggloméré est négligeable. En effet, la variance entre traitements (farine ou agglomérés) reste nettement inférieure à la variance entre répétitions du même dosage. L'agglomération, même à sec, ne paraît donc pas assez brutale pour diminuer significativement la teneur en lysine " disponible " de l'aliment choisi.

b. Glucides.

La teneur en glucides de faible poids moléculaire (méthode de Bertrand) est assez élevée (Io,8 à II,9 p. Ioo de la matière sèche) en raison de la présence dans l'aliment de lait en poudre (lactose) et de sucre (saccharose). On constate (tab1. 5) un parallé-

\section{TABLEAU 5}

Influence du mode de pressage sur les teneurs en glucides de l'aliment

\begin{tabular}{|c|c|c|c|}
\hline & \multicolumn{3}{|c|}{ Mode de pressage } \\
\hline & à l'eau & avec vapeur & à sec \\
\hline \multicolumn{4}{|l|}{$\begin{array}{c}\text { Glucides hydrosolubles totaux } \\
\text { (Bertrand) (\% MS) }\end{array}$} \\
\hline $\begin{array}{l}\text { Farine } \ldots \ldots \ldots \ldots \ldots \ldots \ldots \\
\text { Agglomérés } \ldots \ldots \ldots \ldots \ldots\end{array}$ & $\begin{array}{l}10,9^{a} \\
11,4^{a}\end{array}$ & $\begin{array}{l}11,9^{a 1} \\
11,0^{b 1}\end{array}$ & $\begin{array}{l}10,8^{a 2} \\
10,1^{b 2}\end{array}$ \\
\hline & NS & $P<0,05$ & $\mathrm{P}<0,01$ \\
\hline \multicolumn{4}{|l|}{$\begin{array}{l}\text { Glucides alcoolosolubles } \\
\left(80^{\circ} \mathrm{GL}\right)(\% \mathrm{MS})\end{array}$} \\
\hline $\begin{array}{l}\text { Farine } \ldots \ldots \ldots \cdots \cdots \\
\text { Agglomérés } \ldots \ldots \ldots \ldots \ldots \ldots\end{array}$ & $\begin{array}{l}11,36^{a} \\
11,78^{b}\end{array}$ & $\begin{array}{l}12,08^{a 1} \\
11,57^{a 1}\end{array}$ & $\begin{array}{l}10,87^{a 2} \\
10,95^{a 2}\end{array}$ \\
\hline & $\mathrm{P}<0,05$ & NS* & NS \\
\hline
\end{tabular}

* A la limite de la signification.

$N . B$. : Les valeurs affectées de la même lettre ne sont pas significativement différentes dans une même case.

lisme étroit entre les teneurs observées par les deux méthodes appliquées au dosage des glucides réducteurs totaux ou des glucides alcoolosolubles. Toutefois, les valeurs obtenues par dosage à l'anthrone sulfurique sont toujours plus élevées que celles données par la méthode cuprimétrique de Bertrand. Par ailleurs, quelques contradictions apparaissent en ce qui concerne l'effet de l'agglomération sur la teneur en glucides simples de l'aliment suivant la méthode de dosage. L'agglomération à sec ou à la vapeur semble provoquer une diminution significative de 6,5 à 7,5 p. Ioo des glucides totaux, mais ne paraît pas modifier la fraction alcoolosoluble. Par contre, dans le cas du pressage à l'eau, les teneurs en glucides tendent à augmenter : cette augmentation (5 p. Ioo) est significative pour la fraction soluble dans l'alcool.

I a vitesse d'amylolyse par le stıc pancréatique de porcelet fait apparaittre des différences importantes suivant les traitements (fig. I A). 

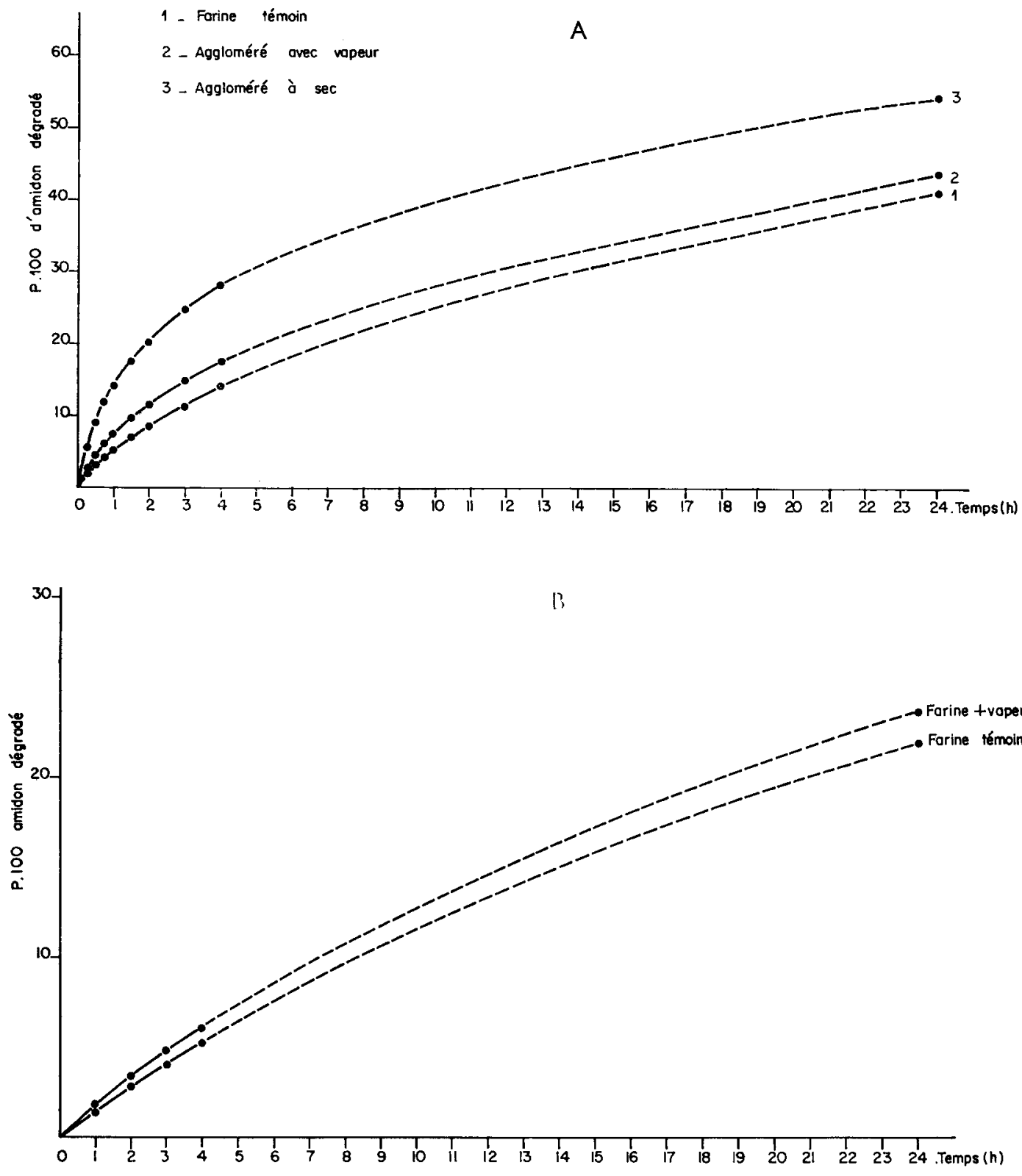

FIG. I. - Hydrolyse de l'amidon de l'aliment

par le suc pancréatique de porcelet en fonction du temps

A : Effet de l'agglomération;

Signification statistique : $3>2>$ I; $\mathrm{P}<0$, oor aux temps I h à $4 \mathrm{~h}$

B : Effet propre de l'injection de vapeur sur la farine

Signification statistique : NS 
L'amidon est d'autant plus sensible à l'amylase qu'il provient d'un aliment ayant subi l'agglomération. Par ailleurs la quantité d'amidon dégradée par unité de temps est toujours supérieure dans le cas du pressage à sec : ce dernier endommage l'amidon beaucoup plus que les autres traitements. L'injection de vapeur en augmentant la vitesse de passage de l'aliment dans la filière $(+44 \mathrm{p}$. roo $)$, réduit l'effet global du traitement sur l'amidon de l'aliment ; dans un dosage ultérieur, nous avons pu montrer que la vapeur est insuffisante pour provoquer à elle seule une augmentation très importante de la vitesse d'amylolyse (fig. I B).

\section{c. Vitamine A.}

L'examen des résultats fait apparaître une action limitée de 1'agglomération vis-à-vis de la vitamine $\mathrm{A}$. Pratiquement nulle dans le cas du pressage à l'eau, la perte atteint 6,6 p. I0O avec la vapeur et II,8 p. I00 à sec, mais n'est significative que dans ce dernier cas.

\section{DISCUSSION}

D'une façon générale, les contrôles analytiques (sauf pour l'amylolyse) effectués sur la farine à l'entrée de la filière et non pas à l'entrée de la presse (conditionneur) ne nous permettent de mettre en évidence que l'influence de la " mise en forme " (agglomération) proprement dite. Cependant, la comparaison entre traitements ne semble pas montrer d'action importante au point de vue biochimique de la seule injection de vapeur sur les composants analysés.

Pour ce qui concerne la lysine disponible, il est difficile de relier nos observations aux données de la bibliographie. La disponibilité de la lysine a été généralement étudiée dans des conditions de laboratoire assez sévères (autoclavage par exemple) et avec des temps de réaction assez longs, afin d'obtenir un blocage appréciable des groupes $\varepsilon$-aminés (OSNER et Johnson, I968). Pourtant Busik (I970) enregistre une perte de 12 à $16 \mathrm{p}$. Ioo de la lysine totale d'un aliment pour porc, aggloméré à l'aide de vapeur (et $5 \mathrm{p}$. Ioo seulement en présence de lignosulfites), mais sans préciser la température atteinte par les produits. En revanche, HuSSAR et ROBBLEE (I962) n'observent aucune différence significative entre la lysine totale ou la lysine disponible de céréales entières et agglomérées dans des conditions voisines des nôtres $\left(72^{\circ} \mathrm{C}\right)$

L'effet du pressage sur la fraction glucidique de faible poids moléculaire doit être interprété avec prudence en raison des nombreux constituants de l'aliment. L'examen des résultats montre que la teneur en glucides simples tend à augmenter lorsque la température reste assez basse (pressage à l'eau) mais tend au contraire à diminuer lorsque la température s'élève (pressage à sec ou avec vapeur). Ceci peut s'expliquer par une transformation due, au pressage, de la fraction amylacée de l'aliment en glucides de faible poids moléculaire, mise en évidence dans le cas du mais par Mercier et Guilbot, I962. En outre, les mêmes auteurs montrent que la céréale possède une activité enzymatique propre qui subsiste même après agglomération. Ces effets conjugués prédomineraient à température modérée. A température plus élevée, une action contraire semble s'exercer par polymérisation des glucides simples 
(caramélisation), ou formation de produits intermédiaires à partir de composés azotés autres que la lysine (SPARK, I969), puisque la " disponibilité " de celle-ci ne paraît pas affectée.

Les résultats relatifs à la vitesse d'amylolyse sont pleinement en accord avec les données de MERCIER et GuILBOT (I962), retrouvés récemment par Aumaitre (I972) pour un aliment de composition voisine aggloméré à sec. De telles modifications de la fraction amylacée (CALE'T, I965) peuvent avoir un effet bénéfique sur l'utilisation digestive des glucides complexes par le jeune animal, notamment le jeune porcelet, qu'il conviendrait de mieux démontrer.

Le pressage à la vapeur n'entraîne pas de perte significative de vitamine $\mathrm{A}$ dans nos conditions expérimentales. En présence d'eau ou de vapeur, l'élévation de température lors du passage dans la filière est moins brutale et l'altération des enrobages de vitamine A s'en trouverait notablement réduite. Les valeurs observées dans le cas du pressage à la vapeur recoupent celles de WORNICK (I959), VALDEBOUZE et LEVY (1972), mais sont beaucoup plus faibles que les chiffres indiqués par BIERER et Vickers (I958), qui mettent en évidence une perte de $32 \mathrm{p}$. Ioo en vitamine $\mathrm{A}$, sans malheureusement préciser les conditions de leur essai. Par contre, dans le cas du pressage à sec, la destruction de vitamine A observée est nettement plus élevée que celle trouvée par VALDEBOUZE et LEVY (I972); elle reste cependant comprise dans les limites données par FRANÇOIs en I960 (o à I9 p. IOO suivant les concentrats de vitamine A utilisés). Cette variabilité des résultats s'explique par la multiplicité des paramètres (PICKFORD, I968; WoRNICK, I959) souvent mal contrôlés qui agissent sur le pressage, paramètres relatifs à la composition de l'aliment et à sa texture, à la nature du concentrat (FRANçors, I960), à la presse (puissance, vitesse de rotation, forme et dimensions de la filière), aux conditions de pressage (température, humidité, débit) et à l'opérateur lui-même (réglages).

En somme, le pressage à l'eau apparaît plus séduisant (minimum d'altérations, moindre consommation d'énergie électrique par tonne fabriquée, caractéristiques physiques satisfaisantes). Cependant, ses inconvénients (conservation du produit fini, moindre état de dégradation de l'amidon) pourraient lui faire préférer le pressage à l'aide de vapeur. Mais les conditions technologiques optima et notamment la diminution de la durée de fabrication observée par l'emploi d'eau ou de vapeur correspondent-elles à une valeur nutritionnelle optimum de 1'aliment chez le porcelet, comme cela a déjà été montré avec du maïs chez le poulet (CALET, I965)? C'est ce qu'il conviendrait de vérifier prochainement.

Rę̧u pour publication en février 1974.

REMERCIEMEN'TS

Nous tenons à remercier ici le personnel de la Fabrique de Mélanges Alimentaires Expérimentaux (I. N. R. A.) où ont été effectués les essais. 


\section{SUMMARY}

\section{EFFECT OF PELIETING CONDITIONS ON SOME PHYSICO-CHEMICAL CHARACTERISTICS OF A PIGLET DIET}

A diet for piglets was pelleted at a semi-industrial scale according to 3 different treatments, $i . e$. dry, with water of with steam. An attempt was made to estimate the changes in some of the dietary components (lysin, carbohydrates, starch and vitamin A) and to determine the physical characteristics of the pellets obtained by means of the different treatments.

Under our experimental conditions, no significant reduction of the "available " fraction of lysin was found. On the other hand, vitamin $\mathrm{A}$ and total reducing carbohydrates as well as the sensitivity of starch towards the amylase breakdown were affected by the hardest conditions (dry pelleting). The combined action of pressure and heat seems to be determining, in particular for starch, whereas heat alone or pressure accompanied by a slight raising of the temperature had almost no effect on the physico-chemical criteria studied.

\section{RÉFÉRENCES BIBLIOGRAPHIQUES}

Adrian J., 1972. La réaction de Maillard vue sous l'angle nutritionnel. I. Domaine de la réaction de Maillard. Ind. alim. agr., 89, I28I-I 289 .

Adrian J., Favier J. C., r96r. La réaction de Maillard. I. Étude du comportement de la lysine pure. Ann. nutr. alim., 15, 181-225.

Alliked J. B., Jensen L. S., McGinnis J., I957. Factors affecting the response of chicks and poults to feed pelleting. Poult. Sci., 36, 517-523.

Aumaitre A., r972. Valeur alimentaire de l'orge dans les rations de sevrage précoce à 2 I jours chez le porcelet. Journées Rech. Porcine en France, Paris, 105-113, I. N. R. A., I. T. P. éd.

Aumaitre A., Salmon-Legagneur E., i96r. Les préférences alimentaires du porcelet. V. Comparaison de divers modes de distribution de l'aliment. Ann. Zootech., 10, I97-203.

Bierer W., Vickers C., r958. The effect of pelleting on vitamins A and E. J. Amer. Vet. Med. Assn, 133, 228.

Braude R., Townsend J. M., Rowel S. G., ig6o. A comparison of meal and pelleted form of creep for suckling pigs. J. A gric. Sci., 54, 274-277.

Busik W., I97o. Influence de la granulation sur la composition chimique et la teneur en acides aminés des aliments composés (en russe). Mukomol'no elev. prom., 1, 23, dans Die Miihle, 110, 202-203.

CALET C., I965. The relative value of pellets versus mash and grain in Poultry Nutrition. World's Poult. Sci. J., 21, 23-52.

CARPENTER K. J., I960. The estimation of the available lysine in animal-protein foods. Biochem., $J ., 7 \%, 604-610$.

Delort-Laval J., Drevet S., r97o. Méthodes d'appréciation de la dureté des fourrages agglomérés. Ind. alim. anim., 213, 43-54.

Drevet S., I97o. Étude de quelques caractéristiques physiques des aliments composés. Bull. Anciens Élèves École Fŗ̧se de Meunerie, 210, 275-285.

Françors A. C., rg60. Étude comparée de la stabilité de la vitamine A dans un composé minéral, un aliment en farine et un aliment aggloméré. Ind. alim. anim., 110, 35-39.

Françors A. C., Pinet A., r96r, Le dosage de l'axérophtol dans les produits alimentaires vitaminisés destinés aux animaux. Ann. Biol. anim. Bioch. Biophys., 1, 258-267.

Friedrich W., I 664 . Zur Technologie des Verpressens von Mischfutter : II. Theoretische Grundlagen zum Pressgranulieren und ihre Folgerungen für die weitere Versuche. Kraftfutter, 47, 352-358.

Guilbot A., Mercier Ch., I962. Répercussions sur la digestibilité de l'amidon des modifications de sa structure physicochimique au cours de ses transformations technologiques. Ind. alim. agric., 79, 939-947.

Hussar N., Robblee A. R., r962. Effects of pelleting on the utilization of feed by the growing chicken. Poult. Sci., 41, I489-I493.

Jensen A. H., I966. Pelleting rations for swine. Feedstuffs. 38, 24-27.

Jensen L. S., Merrill L. H., Reddy C. V., McGinnis J., I962. Observations on eating patterns and rate of food passage of birds fed pelleted and unpelleted diets. Poult. Sci., 41, I4I4-I4I9. 
Lawrence T. L. J., I97I. Cubing the diet of the growing pig : some effects on nutritive value of temperature, pressure and physical form. J. Sci. Food Agric., 22, 403-406.

Maillard L. C., rgi2. Action des acides aminés sur les sucres : formation des mélanoïdines par voie méthodique. C. R. Acad. Sci. Paris, 154, 66-68.

Mercier Ch., I968. Contribution à l'étude de la structure du grain d'amidon au moyen de méthodes physiques et enzymatiques. Thèse doc. d'État, Paris, n ${ }^{\circ}$ C. N. R. S., A. O. 2413, 87 p.

Mercier Ch., Guilbot A., I962. L'influence des conditions de granulation du mais sur les transformations biochimiques de son amidon in vitro et sur sa valeur alimentaire. II. Examen physico-chimique de l'amidon et cinétique de l'alpha-amylolyse in vitro. C. R. Symposium "Céréales et Amylacés ", Paris, I4 novembre.

Moore S., Spackman D. H., Stein W. H., I958. Chromatography of amino-acids on sulfonated polystyrene resins. A nalyt. Chem., 50, I I 85 - I 190 .

Nelson L. F., Catron D. V., 1953. Pigs like pellets. Feedstuffs, 25, I8.

Osner R. C., Johnson R. M., I968. Nutritional changes in proteins during heat processing. J.Fd. Techn., 3, 8I-86.

Prost H. B., Allen, R. N., I963. A standard method of measuring pellet durability. Proc. feed. prod. school, Kansas City, I2-14 nov., 25-29.

Pickford J. R., 1968. The effect of mill processing on vitamin levels in diets. Proc. 2nd Nutr. Conf. Feed Manufact., Nottingham, mars, $175-184$.

Roach A. G., Sanderson P., Williams D. R., I967. Comparison of methods for the determination of available lysine value in animal and vegetable protein sources. J. Sci. Fd. A gric., 18, 274-278.

Salmon-Legagneur F., Aumaitre A., Jounndet C., I968. Étude de quelques facteurs de la stabilité des antibiotiques dans les aliments pour porcelets. Ann. Zootech., 17, 289-305.

Salmon-Legagneur E., Fevrier R., I955. Les préférences alimentaires du porcelet. I. Influence du mode de présentation des aliments : granulés ou farine. Ann. Zootech., 4, 215-218.

Schultz R., 1965. Uber das Messen der mechanischen Festigkeit von gepressten Mischfutter. Die Mïhle, 102, 147-I55.

Spark A. A., 1969. Role of amino acids in non enzyme browning. J. Sci. Fd. Agric., 20, 308-3i6.

Stockstad E. L. R., Esposito R. G., Grady J. E., Williams W. L., I952. Stability of antibiotics in poultry feeds during pelleting and storage. Poult. Sci., 31, 46-68.

Valdebouze P., Levy B. R., I972. Influence de l'agglomération et du stockage des aliments composés sur leur teneur en vitamine A. Ind. alim. anim., 2, 49-54.

Vanschoubroek F., Coucke L., Van Spaendonck R., I97I. The quantitative effect of pelleting feed on the performance of piglets and fattening pigs. Nutr. Abst. Rev., 41, I-9.

WorNick R. C., 1959. What really happens to micro-ingredients in pelleting? Feed pelleting and its effects on micro-ingredients. Proc. Feed Prod. School, Kansas City, 180-198.

Wornick R. C., I968. Stability of micro-ingredients in Animal Feed Products. Feedstuffs, 40, 25-3I. 